Research Article

\title{
Application of Basketball Training System Based on Dynamic Intelligent Fog Computing Network
}

\author{
Ke Yang (1D \\ Physical Education Department, Xihua University, Chengdu 610039, Sichuan, China \\ Correspondence should be addressed to Ke Yang; 0120030089@mail.xhu.edu.cn
}

Received 10 June 2021; Revised 6 July 2021; Accepted 3 August 2021; Published 13 August 2021

Academic Editor: Sang-Bing Tsai

Copyright (c) $2021 \mathrm{Ke}$ Yang. This is an open access article distributed under the Creative Commons Attribution License, which permits unrestricted use, distribution, and reproduction in any medium, provided the original work is properly cited.

\begin{abstract}
Although the development of the mobile Internet and the Internet of Things has greatly promoted the progress and development of society, it has also created many problems for people on the road of scientific and technological exploration. In order to meet the problems and requirements of high bandwidth, high load, and low latency in the current network development, the emergence of the concept of mobile edge computing has attracted extensive attention from the academic community. This article focuses on the representative mode of mobile edge computing-fog computing (in this model, data, (data)processing, and applications are concentrated in devices at the edge of the network, instead of being stored almost entirely in the cloud). By applying it to the development and operation of basketball training system, it explores the performance of dynamic intelligent fog computing in intelligent end user services. This paper proposes a fog resource scheduling scheme based on linear weighted genetic algorithm, which converts the problem of multiobjective optimization into a single-objective optimization problem. When applying the genetic algorithm based on weighted sum, preference is given to delay, communication load, and service cost. Value is integrated into an objective function to perform genetic operations to get a better solution. From the experimental data, the system can support 20 DCTU terminals with a pressure request of 10 messages per second per terminal under the pressure environment created by the pressure test input data. The barrier-free transmission distance is $200 \mathrm{~m}$, and the barrier transmission distance is $50 \mathrm{~m}$. It has strong fault tolerance.
\end{abstract}

\section{Introduction}

The centralized big data processing architecture centered on the cloud computing model is a commonly used technology in the modern computer field, but at the same time, due to its own characteristics, this computing architecture cannot be extended to all fields. The final result of the Internet of Things is the connection of any individual mobile terminal. Only fog computing can carry such a large amount of calculation and data.

In foreign countries, fog computing, as a new computing model, has become an important research direction for many scholars. Fouquet has conducted research on energy management as a service based on fog computing platform. Energy management can also help people achieve zero net energy in the residential sector. Due to the continuous increase in the number of sensors and actuators, a powerful computing model is needed as an auxiliary means. Considering that the performance of cloud computing in terms of scalability is prone to potential hidden dangers, he applied fog computing to a new type of energy management platform in his research. From the results of simulation experiments, the platform assisted by fog computing has good scalability and adaptability, and users can realize energy management through customized control as a service. Of course, if the fog computing platform is to be promoted on a large scale, more experimental data is needed [1]. Rahmani has conducted research on smart electronic health gateways at the edge of the healthcare IoT based on fog computing. In most healthcare systems based on the Internet of Things, especially in smart homes or hospitals, bridging points (i.e., gateways) are required between the sensor infrastructure network and the Internet. Rahmani and his team use the gateway's strategic position at the edge of the network, 
providing higher-level services such as local storage, realtime local data processing, and embedded data mining, to form an intelligent electronic health gateway. From the experimental results, although the medical system still has room for optimization in terms of resource allocation efficiency, it has already demonstrated a good dynamic optimization effect [2].

In recent years, domestic research on dynamic intelligent fog computing has also gained more and more results. Peng conducted research on the problems and challenges in the wireless access network based on fog computing. In his research, he introduced a radio access network based on fog computing as an example of the fifth-generation wireless communication system that is expected to provide high frequency spectrum and energy efficiency. Making full use of the advantages of local radio signal processing, cooperative radio resource management, and distributed storage functions in edge devices can reduce the heavy burden of fronthaul and avoid large-scale radio signal processing in a centralized baseband unit pool. From the experimental analysis, the system architecture and key technologies mentioned in the research have very good performance in optimizing the transmission mode and improving the antiinterference suppression, but they are not enough to solve some complex problems in network function virtualization [3].

Based on the dynamic intelligent fog computing network, this paper conducts in-depth research on the development of basketball training system. The research mainly starts from the following parts: first, this article introduces the design to technology and methods in system development, including dynamic intelligent fog computing network, system architecture design based on fog computing, system resource management based on fog computing, and genetic algorithm based on linear weighting. Secondly, this article has conducted demand analysis and technical design around the basketball training system. Finally, this paper conducted an experimental test on the system and demonstrated the effect of fog computing in system development and application through observation.

Compared with the traditional basketball training method, the method introduced in this article is much more intelligent.

\section{Technology and Method of Basketball Training System Based on Fog Calculation}

2.1. Dynamic Smart Fog Computing Network. In order to effectively solve the high network load, high bandwidth, low latency, and other requirements brought about by the rapid development of the mobile Internet and the Internet of Things, the concept of mobile edge computing has been proposed and has received extensive attention from academia and industry [4]. Fog computing is an edge computing concept. Although it is an extension of cloud computing, fog computing is closer to the edge network than highly concentrated cloud computing, with wider geographic distribution, higher real-time performance, and low latency [5]. Therefore, if it is for some delay-sensitive applications, fog computing will be more suitable than cloud computing. Compared with sensor nodes, fog computing nodes have certain storage capabilities and data processing capabilities and can simply process some data and perform some simple applications, especially some applications based on geographic location [6].

According to the characteristics of edge computing, the overall system function based on the collaboration of edge devices and cloud computing centers can be realized. The main requirements of the data collection and analysis system include data collection function, data preprocessing function, data analysis function, and internal communication of the system. Whether in industrial production or daily life, a large amount of data information is mainly collected through various sensors $[7,8]$. With the continuous improvement of social informatization, the demand for data collection from such sources is also increasing.

\subsection{System Architecture Design Based on Fog Computing.} The fog computing simulation system mainly includes three parts: the cloud data center, the fog computing layer, and the terminal application of the Internet of Things. The cloud data center is mainly responsible for communicating with the fog layer and processing large data tasks. The terminal nodes mainly realize data collection and release to the fog layer. In the function of the task, the fog layer is a cluster of nodes with computing capabilities, which mainly include the leader election module, the energy module, and the heartbeat detection module to ensure the normal operation of the fog layer decentralized computing node system [9]. The fog layer is responsible for communication with cloud data centers and terminal applications, as well as task processing for small data volumes. Figure 1 is a flowchart of the fog computing simulation system.

In the start-up stage before the system is officially running, the computing node cluster of the fog layer must first be warmed up, and the leader node is elected to collect and summarize the information of the fog layer node and undertake the communication between the cloud data center and the terminal application node during the system operation-communication task $[10,11]$. The UID value represents the current stability and resource size of the node. The stability of the node is measured by the available time in the standby state of the node, and the available resources of the node are measured by the storage capacity and data processing speed of the node. Before the system is officially running, the UID of the node value satisfies the following formula:

$$
\begin{aligned}
\text { node_UID } & =t_{-} \text {run }+ \text { process_rate }+ \text { storage }, \\
R\left(S_{i}\right) & =\left\{s_{j} \in N \mid a i j=1\right\} .
\end{aligned}
$$

After the node goes online, the corresponding UID value is

$$
\text { node_UID }=\frac{\left(E_{\text {start }}-E_{\text {thre }}\right)}{E_{\text {standby }}}+\text { process_rate }+ \text { storage } .
$$






FIgURE 1: Flow chart of fog computing simulation system.

The fog layer computing node is a decentralized design, and the functions and corresponding resources of the leader node and other ordinary nodes are equal and can be transformed into each other. During the formal operation of the basketball training system, computing nodes may withdraw from the fog computing system due to node failures and link failures. In order to cope with the impact of leader node failures in the system on the fog computing system services, it quickly discovers the failure of the leader node and elects a new leader node to replace the original node to undertake the task of system node equipment operation monitoring and resource allocation $[12,13]$.

\subsection{System Resource Management Based on Fog Computing.} In the fog node layer of system resource management based on fog computing, each agent node manages a fog node cluster, and the agent node effectively manages the fog node resources in the cluster $[14,15]$. Set the value of $m$ to the number of all leaf nodes in the structure graph, $n$ to the number of leaf nodes directly or indirectly verified by a leaf node, and $T$ to the total number of times the fog node participates in providing services. The (feasibility) reputation value of the fog point in the structure diagram is

$$
\begin{aligned}
\text { rep } & =\frac{n}{m} * 100 \%, \\
\mathrm{REP} & =\sum_{i=1}^{T} \frac{\mathrm{rep}_{i}}{T} .
\end{aligned}
$$

Because the computing resources of the fog node are limited, a user's task request can be divided into multiple subtasks to complete. These subtasks have a front and back constraint relationship, which can be understood as an Internet of Things service, which can be broken down into multiple microservices to achieve $[16,17]$. Set $T$ as a task collection, $E$ represents the relationship between tasks, and $e_{i j}$ represents the amount of communication that task $T_{i}$ needs to transmit data to task $T_{j}$. Then they satisfy the following formula:

$$
E=\left[\begin{array}{ccc}
e_{11} & \cdots & e_{1 n} \\
\vdots & \ddots & \vdots \\
e_{n 1} & \cdots & e_{n n}
\end{array}\right]
$$

It is known that $F_{i}$ represents the $i$ th fog node in the fog node set, $d$ is the number of resource attributes, $p_{k}$ is the value of the $k$ th attribute, and $f_{k}$ is the weight of the $k$ th attribute. Then there is the following formula:

$$
D=\left[\begin{array}{ccc}
d_{11} & \cdots & d_{1 m} \\
\vdots & \ddots & \vdots \\
e_{m 1} & \cdots & e_{m m}
\end{array}\right]
$$

The calculation formula of resource attribute value is

$$
\rho=\frac{\sum_{k=1}^{d} \rho_{k} * f_{k}}{\sum_{k=1}^{d} f_{k}} .
$$

Since the performance of fog node equipment is different, the computing power of the fog cluster can be measured by calculating the average processing rate $\bar{\rho}$ of the fog cluster node. Let $m$ be the total number of fog nodes in the fog cluster; then the rate formula meets

$$
\bar{\rho}=\frac{\sum_{i=1}^{m} \rho\left(F_{i}\right)}{m} .
$$

The scheduling strategy in this paper is represented by two sets of sequences: one set is the task execution sequence and the other set is the fog node sequence corresponding to the execution task [18]. Because the model given by the task is in the form of a directed acyclic graph, a priority must be defined for each subtask to generate a sequence of task execution order.

\subsection{Genetic Algorithm Based on Linear Weighting}

2.4.1. Genetic Algorithm. This paper improves the basis of the basic genetic algorithm, uses a linear weighting method to merge multiple targets into one target, and assigns a 
different weight to each target, which is set according to the degree of preference requested by the user [19]. For the three optimization goals proposed in this paper, the three goals are first normalized, and then the fitness function is established according to the preference weight, so that multiple optimization goals are combined into one objective function [20]. Among them, $\alpha, \beta, \gamma$ are the optimization weights of the three objectives. The fitness function in this paper is defined as $f$; then they satisfy the following formula:

$$
f=\sum_{i=1}^{s}\left(\alpha * \frac{\operatorname{Delay}(i)}{S * \text { Delay }}+\beta * \frac{C \text { Load }(i)}{S * \text { CLoad }}+\gamma * \frac{\text { Con }(i)}{S * \text { Con }}\right) .
$$

In the population, genetic operators need to go through three processes of selection, crossover, and mutation to complete genetic operations [21]. For selection operator, this article chooses the way of roulette. After roulette selection, individuals with larger fitness values in the population are more likely to be selected, which ensures that they have excellent genes. The specific selection method is as follows:

$$
\begin{aligned}
& p(i)=\frac{f(i)}{\sum_{i=1}^{S} f(i)}, \\
& Q(i)=\sum_{j=1}^{i} p .
\end{aligned}
$$

This article adopts the traditional single-point crossover method. A crossover point is set between any two adjacent loci in each chromosome sequence, and one crossover point is selected arbitrarily in the first gene locus, and then swap out all the genes behind that intersection. In the mutation operator, each chromosome sequence uses basic bit mutation, randomly selects one of the $n$-bit gene positions, and replaces it with a random number in between, thus generating a new chromosome, ensuring the diversity of the population.

2.4.2. Improved Genetic Algorithm. The genetic algorithm based on linear weighting has a good performance in meeting the needs of users for different optimization target preferences. With the gradual increase in the number of users, the scheduling of fog computing resources must also meet the needs of general users. There are no special requirements for communication load and service cost, and at this time, it is necessary to have a certain optimization effect for each target. To this end, this paper proposes a nondominated selection genetic algorithm (NSGA-II) for multiobjective optimization and has made certain improvements to it.

This paper establishes three fitness functions, which represent the normalization of the three goals of delay, communication load, and service cost. They correspond to the following formulas:

$$
\begin{aligned}
& f_{i}=\sum_{i=1}^{s} \frac{\text { Delay }(i)}{S * \text { Delay }} \\
& f_{2}=\sum_{i=1}^{S} \frac{\text { CLoad }(i)}{S * \text { CLoad }} \\
& f_{3}=\sum_{i=1}^{S} \frac{\text { Con }(i)}{S * \text { Con }}
\end{aligned}
$$

There may be special individuals in the initial population with abnormal fitness values. In order to prevent it from affecting the development direction of the entire population, the algorithm converges to the local optimal solution. In the later stage of evolution, when the genetic algorithm gradually converges, it is difficult to continue to optimize the selection due to the closer fitness of the population individuals, resulting in uneven distribution of the population individuals. At this time, the individual fitness value should be increased to improve the selection ability. Based on this, this article establishes a formula for fitness value calibration:

$$
f^{\prime}=\frac{1}{f_{\min }+f_{\max }}\left(f+f_{\min }\right) .
$$

In the process of evolution, in order to make the worst individuals change faster, this article sets the crossover rate and mutation rate of these individuals to the maximum within the conditional range, and the crossover rate and mutation rate of the dominating set individuals are based on the hierarchical relationship which changes linearly. Knowing that $p_{c}^{\prime}$ is the crossover rate of the population in the next iteration, $p_{c \mathrm{Max}}$ is the maximum crossover rate, and $p_{b}^{\prime}$ is the mutation rate of the population in the next iteration, the model calculation formulas for the crossover rate and mutation rate in the population are

$$
\begin{aligned}
& p_{c}^{\prime}=\frac{\left(p_{c \mathrm{Max}}-p_{c}\right)\left(i_{\mathrm{rank}}-1\right)}{K-1}+p_{c}, \\
& p_{b}^{\prime}=\frac{\left(p_{b \mathrm{Max}}-p_{c}\right)\left(i_{\mathrm{rank}}-1\right)}{K-1}+p_{b} .
\end{aligned}
$$

In the early stage of algorithm iteration, the global space needs to be searched to improve population diversity, and a smaller cross-distribution index and variation distribution index should be used. As the number of iterations increases, the algorithm should gradually enhance the local search to obtain the local optimal solution.

\section{Basketball Training System Experiment Based on Dynamic Intelligent Fog Computing Network}

3.1. Experimental Background. Basketball has always been one of the most popular sports in our country. From a macroperspective, it can be the sports reform promoted in colleges and universities, the national sports advocated by the general public, or professional basketball competitions that continue to rise in popularity, which reflects the 
country's encouraging attitude towards the scientific movement. System science theory believes that all organizations can be regarded as subsystems in a large system, and the process of management is an optimal process; that is, system optimization is achieved through rational allocation of organizational resources. This article regards basketball training as a project, hoping to realize a common comprehensive management with athletes' physical training, athletes' professional skills training, and scientific competitions. To this end, this article introduces the concept of fog computing, hoping to develop a basketball training system through dynamic intelligent computing to provide more technical support for promoting sustainable basketball training.

The research object of this article is 44 students in a certain professional physical education elective basketball class of University $A$.

\subsection{Experimental System Development. The experimental} system of this article is composed of four parts: data acquisition terminal DCTU, acquisition and calculation server DCS, database DB, and data analysis server DAS. Among them, DCTU completes database collection and reporting through sensors, DAS is responsible for calculating and storing data according to the calculation model, and DAS is responsible for analyzing data and realizing the business in demand. DAS is also responsible for basic configuration and configuration management.

Before the formal system development, it is necessary to conduct a demand analysis of the task objectives. In order to achieve scientific basketball training management, this article believes that the system should have athlete information management, athletes' personal training directions and plans, competition information management, and so on. Compared with the traditional training teaching plan, this article hopes that the system will have stronger professionalism, intelligence, and one-to-one personalized recommendation.

Physical fitness is an abstract concept without a unified definition and theoretical guidance. How to select the physical fitness index is a key concern. If the indicators are too complex, it is impossible to construct a collector to collect the corresponding data; but the lack of indicators will also cause the system to lose its reference value. If the system wants to produce effective training suggestions, it must fully absorb the training management experience and training theory of coaches and researchers on the basis of analyzing athletes' physical fitness data and provide a valuable graphical information interface. Figure 2 is the process of judging the direction of basketball training for athletes.

It can be seen from Figure 2 that, in order to realize dynamic intelligent management with the help of fog computing in the system, it is necessary to process and analyze various index data on the basis of mastering the basic information of athletes. When athletes train according to the system recommended training program, they should also monitor their physical indicators in time and use this as a basis to judge whether training methods or training intensity need to be adjusted.
3.3. Experimental System Test. On the basis of demand analysis, this article has completed the simulation development of the system. Before the formal experimental test, the system performance needs to be tested. The key content of this test includes (1) how to generate a large amount of sports data for delivery, collection and calculation, server processing, and business analysis systems for analysis; (2) the idea that the barrier-free effective distance between the collection terminal and the DCS should not be less than 200 meters, and there should be no obstacles (less than 50 meters); (3) whether the collection and calculation server has sufficient data throughput and fault tolerance.

\section{Application Research of Basketball Training System Based on Dynamic Intelligent Fog Computing Network}

The youth stage is a critical period for cultivating athletes' basketball hobbies. In order to better reflect the help of basketball training system to basketball training teaching, this article investigates the factors restricting the improvement of the team's training level. Figure 3 shows the results of the survey.

It can be seen from Figure 3 that, among the various factors affecting basketball training level, the restriction caused by insufficient training level of coaches accounted for up to $24 \%$, which is enough to prove how good coaches and suitable training programs can improve the professional level of athletes, which is important. In order to help athletes find the most suitable basketball training program, this article will develop a basketball training system based on the dynamic intelligent fog computing network and realize scientific training plan formulation by collecting, processing, and analyzing various indicator data of athletes.

\subsection{Performance Test of Smart Basketball Training System} Based on Fog Computing. In order to better reflect the test effect, this paper makes a fictitious DCTU in the system, which is used to generate massive exercise data for processing and analysis. It also simulates receiving instructions and sending data messages to DCS. Table 1 shows the stress test cases of the basketball training system.

DCTU is direct connection to the test unit.

For the test focus of the effective distance, the barrierfree DCTU test can arrange several DCTUs in an open area and test according to the load requirements of PTC-001. For the obstacle DCTU test, several DCTUs can be arranged in the open area and the human body barrier can be appropriately increased. According to PTC-001 load requires testing. Table 2 and Figure 4 are the pressure test data of the basketball training system.

It can be seen from Table 2 and Figure 4 that the system in this article believes that the system can support 20 DCTU terminals with a pressure request of 10 messages per second per terminal under the pressure environment created by the pressure test input data. The barrier-free transmission distance is $200 \mathrm{~m}$, and the barrier transmission distance is $50 \mathrm{~m}$. 




FiguRE 2: Judgment process of athlete basketball training direction.

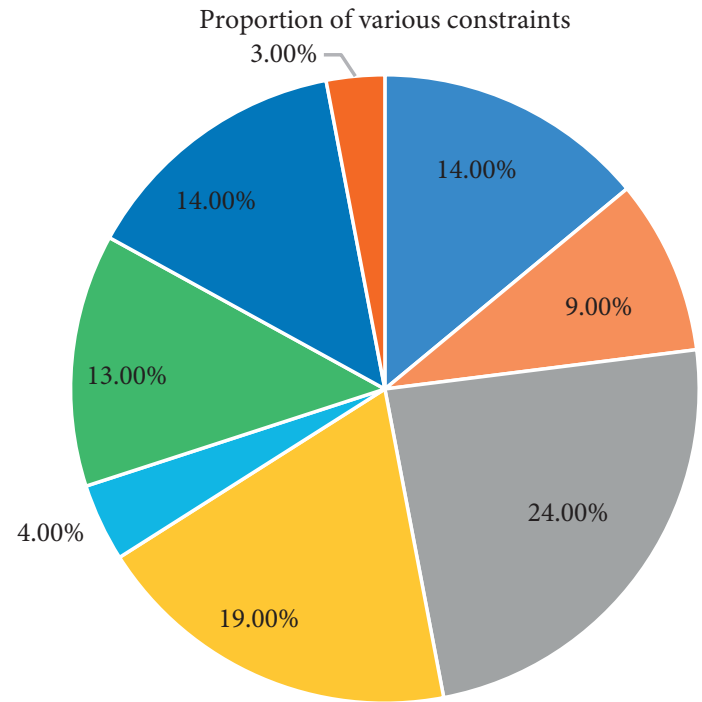

Lack of policy guarantee

Parents do not support

Inadequate training level of coaches

Contradiction between learning and training

Venue facility restrictions

Insufficient capital investment

Limited admissions channels

Other

FIGURE 3: Factors restricting the improvement of team training.

It has strong fault tolerance. The test results show that the system is qualified and meets the established requirements.

4.2. Analysis of Physical Fitness Data in Intelligent Basketball Training System. The physical data used in this system are all completed by a personal collector designed and placed on the athlete. During daily training and competitions, athletes can complete assessment data including body temperature $(T)$, heartbeat $(B)$, acceleration vector $(A)$, and various professional tests. Since basketball players are always exercising vigorously, they have higher requirements on the physical and biological characteristics of the terminal. First of all, the terminal and supporting sensors need to carry body parts that are basically inaccessible between athletes and that are consistent with the torso motion vector. Secondly, it has strong earthquake resistance; thirdly, it is tightly carried and not easy to fall and shift. Due to the built-in gyroscope, the device does not require the current posture. In addition, for the external heartbeat (wrist or neck) sensor, it needs to have good biological characteristics, such as being moisture-proof, nontoxic, and soft, and a reliable transmission line to connect to the collector.

The physical processing data is mainly based on the acceleration vector $A$ (the ratio of the amount of speed change to the time it takes for this change to occur). After the raw data is collected wirelessly, it is calculated in real time by the data collection server. Table 3 is a processing data table based on the original data. Figure 5 shows the comparison of the effectiveness in the system after data processing.

Vertical height is height from bottom to top on the plane.

It can be seen from Figure 5 that the effectiveness of the processed data in the basketball training system has increased from $73.71 \%$ to $90.86 \%$. The various physical data of athletes are not only diverse in variety, but also extremely fast, and it is difficult for the system to formulate reasonable training programs for athletes through the collected raw data. The data after scientific processing is more in line with the calculation model of machine intelligence, so it can effectively improve the professionalism of the system training program and provide data support for the establishment of new physical indicators. Table 4 shows the physical fitness index based on processing data. Figure 6 shows the recognition of the new physical fitness index by the professional coaching team.

It can be seen from Figure 6 that the professional coaching team has a high degree of recognition of the new physical fitness indicators, among which the professional recognition of the mileage indicator is as high as 90.6. On 
TABLE 1: Stress test case of basketball training system.

\begin{tabular}{|c|c|c|c|}
\hline Number & PTC-001 & Number & PTC-002 \\
\hline Test target & $\begin{array}{c}\text { Higher load testing, } 100 \% \text { system availability must } \\
\text { be provided }\end{array}$ & Test target & $\begin{array}{l}\text { Ultrahigh load testing requires } 100 \% \text { system } \\
\text { availability }\end{array}$ \\
\hline $\begin{array}{l}\text { Testing } \\
\text { method }\end{array}$ & $\begin{array}{c}1-N \text { virtual DCTU send data packets to DCS at a } \\
\text { certain frequency }\end{array}$ & $\begin{array}{l}\text { Testing } \\
\text { method }\end{array}$ & $\begin{array}{c}1-N \text { virtual DCTU send data packets to DCS at a } \\
\text { certain frequency }\end{array}$ \\
\hline Input data & $\begin{array}{l}20 \text { DCTU (2 games at the same time), } 10 \text { packets/ } \\
\text { sec/DCTU }\end{array}$ & Input data & $\begin{array}{l}50 \text { DCTU (3 games at the same time), } 10 \text { packets } / \mathrm{sec} / \\
\text { DCTU }\end{array}$ \\
\hline $\begin{array}{l}\text { Expected } \\
\text { results }\end{array}$ & $\begin{array}{c}\text { Provide } 100 \% \text { system availability, } 100 \% \text { business } \\
\text { accuracy, high system load }\end{array}$ & $\begin{array}{l}\text { Expected } \\
\text { results }\end{array}$ & $\begin{array}{l}\text { Provide } 90 \% \text { system availability, } 100 \% \text { business } \\
\text { accuracy, ultrahigh system load }\end{array}$ \\
\hline
\end{tabular}

TABLE 2: Basketball training system stress test data.

\begin{tabular}{lcccc}
\hline & Packet loss rate (\%) & Data integrity (\%) & CPU load (\%) & Database disk (\%) \\
\hline PTC-001 & 0 & 100 & 35 & 25 \\
PTC-002 & 8 & 100 & 95 & 40 \\
PTC-003 & 0 & 100 & 20 & 12 \\
PTC-004 & 10 & 100 & 25 & 20 \\
\hline
\end{tabular}

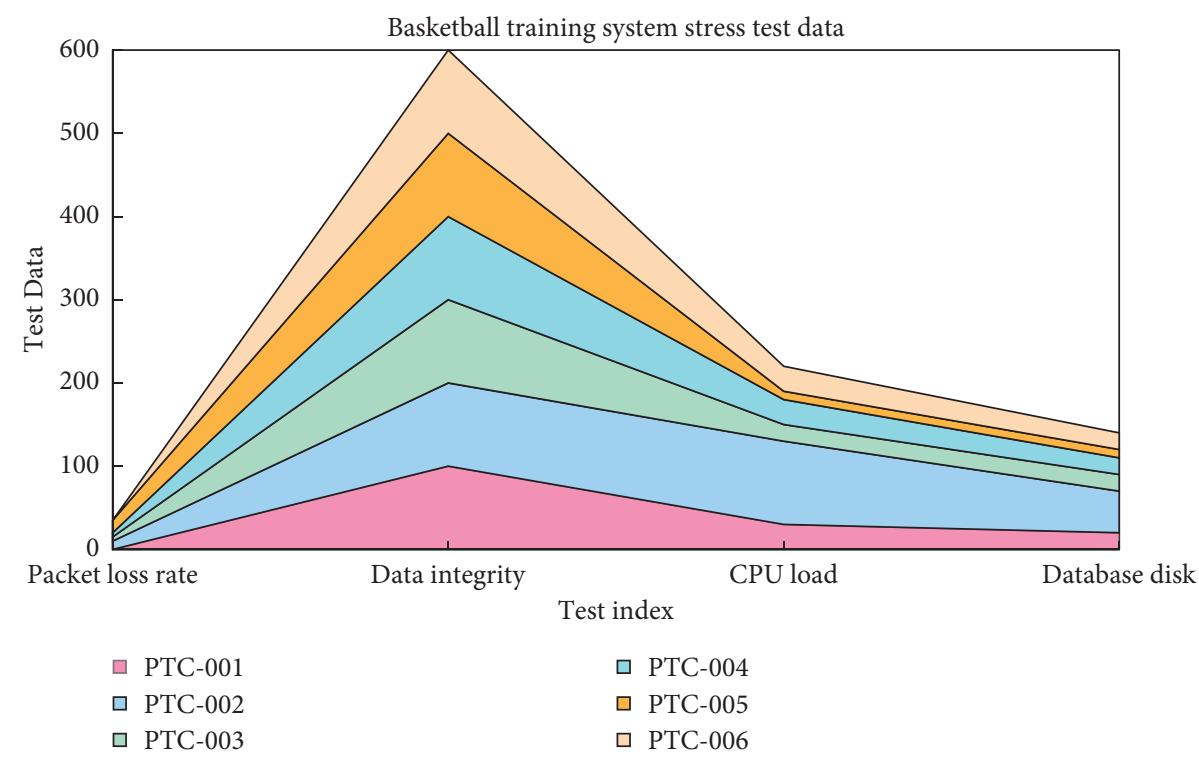

Figure 4: Basketball training system stress test data.

TABle 3: Processing data table based on raw data.

\begin{tabular}{lcc}
\hline Name & Symbol & Meaning \\
\hline Vertical acceleration vector & $A v$ & The vertical component of $A$ calculated from $A$ \\
Horizontal acceleration vector & $A h$ & The horizontal component of $A$ calculated by $A$ \\
Horizontal velocity vector & $V h$ & Current horizontal velocity calculated using $A h$ \\
Vertical velocity vector & $V v$ & Current vertical speed calculated using $A h$ \\
Vertical height & $H$ & Current altitude using $A v$ points \\
Running mileage & $S$ & Current mileage using $A h$ points \\
High jump & $H j$ & The maximum height reached by a jump \\
\hline
\end{tabular}

this basis, the coaching team fine-tuned the physical fitness indicators based on the actual situation. Among them, the total physical fitness index is obtained after a comprehensive calculation of speed index, acceleration index, mileage index, high jump index, and long jump index.
In addition to conventional physical fitness indicators, the system also uses dynamic intelligent fog to calculate some advanced indicators that affect basketball training plans, including role competence indicators, role potential indicators, and training effect indicators. For example, 


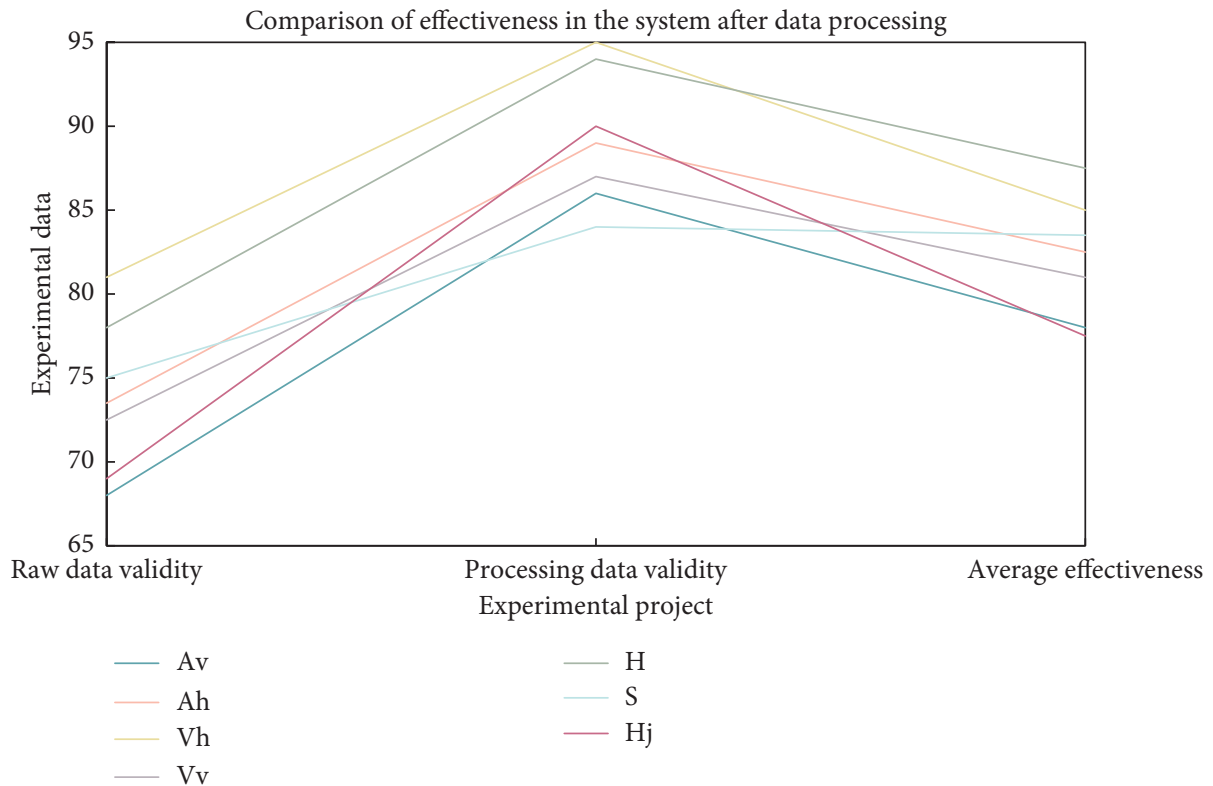

FIGURE 5: Comparison of effectiveness in the system after data processing.

Table 4: Physical fitness index based on processing data.

\begin{tabular}{|c|c|c|}
\hline Category & ymbol & Meaning \\
\hline Speed indicator & Iv & Comprehensive index of average speed and maximur \\
\hline Accel & Ia & $\begin{array}{l}\text { Comprehensive index of average acceleration and maximum acceleration calculated by acceleration } \\
\text { sequence }\end{array}$ \\
\hline Milea & Is & $\begin{array}{c}\text { Comprehensive indicators of average mileage per second and maximum mileage calculated through mileage } \\
\text { sequence }\end{array}$ \\
\hline Hig & $I h$ & Comprehensive index of average height and $n$ \\
\hline Long & $I j$ & Comprehen \\
\hline $\begin{array}{l}\text { Physical fitness } \\
\text { index }\end{array}$ & $P$ & $\begin{array}{l}\text { Comprehensive index of average physical energy consumption and maximum physical energy consumption } \\
\text { calculated by physical energy sequence }\end{array}$ \\
\hline
\end{tabular}
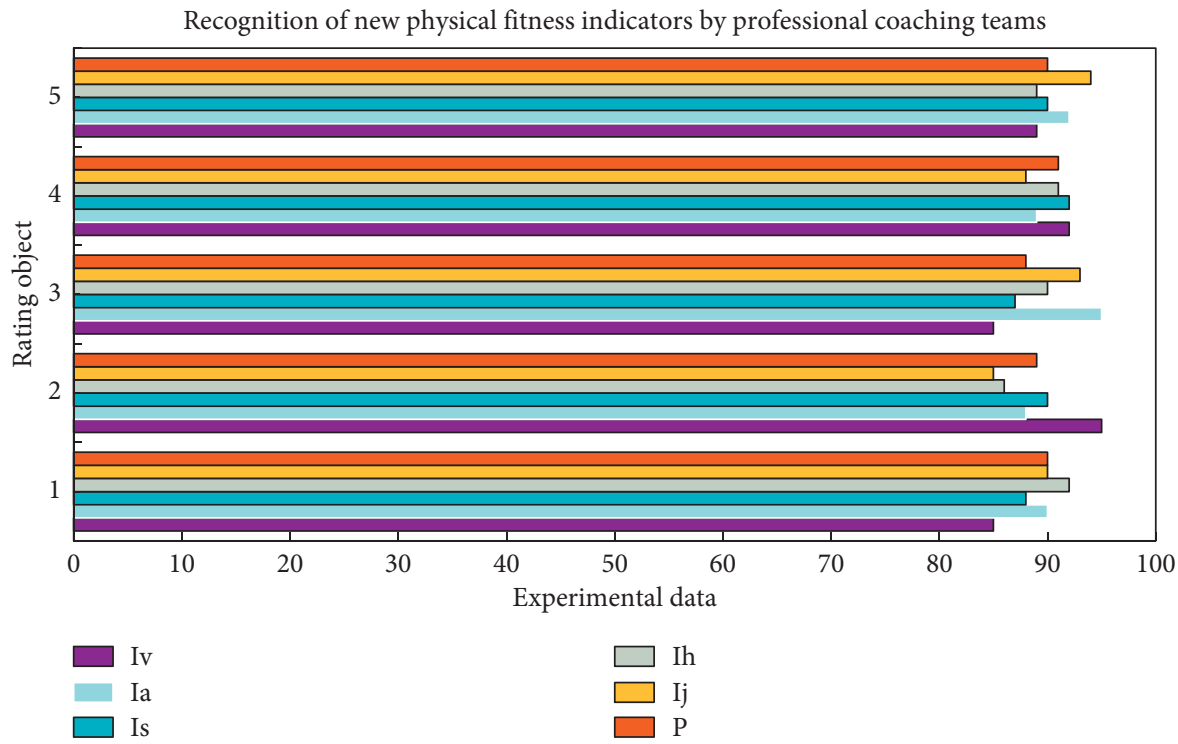

FIgURE 6: Recognition of new physical fitness indicators by professional coaching teams. 
TABLE 5: Simulation parameter setting table.

\begin{tabular}{lc}
\hline Parameter & Range \\
\hline Number of tasks & 20,200 \\
Task calculation & $5,100 \mathrm{MB}$ \\
Task traffic & $5,100 \mathrm{MB}$ \\
Nonforward nodes required by the task & $5.50 \mathrm{MB}$ \\
Number of fog nodes & 6.10 \\
Fog node processing rate & $5.20 \mathrm{MB} / \mathrm{sec}$ \\
Fog node transmission rate & $50,200 \mathrm{MBps}$ \\
Fog node resource calculation unit price & $2.5 \mathrm{cent} / \mathrm{sec}$ \\
\hline
\end{tabular}

coaches believe that athletes with forward potential qualities need to have high qualities in terms of average speed improvement rate, maximum speed, maximum jumping, and average physical improvement rate. Then the system will comprehensively analyze the data of all athletes in the database, determine each person's role competence indicators, and then work out a suitable basketball training plan.

\subsection{Realization of Fog Computing Basketball Training System.} In order to reflect the effect of fog computing in resource management and scheduling in the basketball training system, as well as the effect of the improved nondominant selection genetic algorithm in system optimization, this paper sets the simulation parameters and analyzes the simulation results. Table 5 is the simulation parameter setting table.

The simulation tool of this article is MATLAB R2016b. It is simulation software. In the experiment, this paper gives a task graph of the constraint relationship and a random network topology graph with the number of fog nodes ranging from 6 to 10 . The average node degree of the network topology graph is set to 3 , and the connection probability between nodes is 0.5 . It can be seen from Table 5 that the parameters of this experiment are set as follows: the range of task number is $[20,200]$, the range of task calculation is $[5,100] \mathrm{MB}$, the range of task communication is $[5$, $100] \mathrm{MB}$, the task requires nonforward node, and the data range is $[5,50] \mathrm{MB}$.

In this paper, the population size is set to 100 , the maximum number of iterations is set to 300 , the crossover probability is set to 0.85 , the mutation probability is set to 0.1 , the number of tasks is set to 30 , and the number of fog nodes is 8 . The time delay in the simulation results, the communication load, and service cost are the average values after 30 experiments. Figure 7 shows the weight changes of the three types of optimization objectives.

It can be seen from Figure 7 that the optimization effects of the three objectives are better as the preference weight increases, which can satisfy service requests with different degrees of preference. When the weight of the two goals, time delay and service cost, is about 0.4 , the optimization effect of the goal will tend to stabilize, and the weight of the communication load goal will start to stabilize when the weight is
0.6. From this analysis, it can be concluded that users can appropriately increase the weight of other goals according to their own needs, while ensuring that their preferences are met, to ensure that the overall service quality is met.

In order to analyze the impact of changes in the number of tasks on the target optimization weight, this paper sets the population size to 100 , the maximum number of iterations to 100 , the number of fog nodes to 7 , the crossover probability to 0.85 , and the mutation probability to 0.1 , among which three target values are the average value after 30 experiments. In order to compare the three objectives to obtain the optimized results, this paper sets the optimization weight of delay and service cost to 0.3 and the optimization weight of communication load to 0.4 . Figure 8 shows the numerical changes of the three goals as the number of tasks continues to increase.

It can be seen from Figure 8 that when optimizing the two goals of delay and communication load, the genetic algorithm proposed in this paper has a better optimization effect than the Min algorithm as the number of tasks continues to increase while optimizing service costs. This goal does not perform better to optimize performance. But generally speaking, the genetic algorithm based on linear weighting proposed in this paper has better optimization performance than the locally better Min algorithm. At the same time, it can be concluded from the above analysis that this genetic algorithm is more suitable for users to prefer a certain target and can obtain a good optimization effect. For general user requirements, if you want to obtain an overall better service quality, genetic algorithms cannot show obvious advantages. For this reason, this paper improves the genetic algorithm (GA) based on linear weighting and optimizes it to the nondominated selection genetic algorithm (NSGA). Figure 9 shows the comparison of the number of iterations of the three genetic algorithms.

Set the maximum number of iterations to 300 , the number of populations to 150 , and the number of tasks to 30 . The other settings are the same as the above parameters. It can be seen from Figure 9 that the average value of the crowded distance between two adjacent solutions in the Pareto solution set obtained by the NSGA is smaller than the traditional GA and the Min algorithm, which shows that the NSGA is in the fog computing environment. The nondominated solution set has better distribution. 




_ Time delay A

- Communication load A

_ Time delay B

- Service fee A

- Communication load B

Figure 7: Weight changes of the three types of optimization goals.

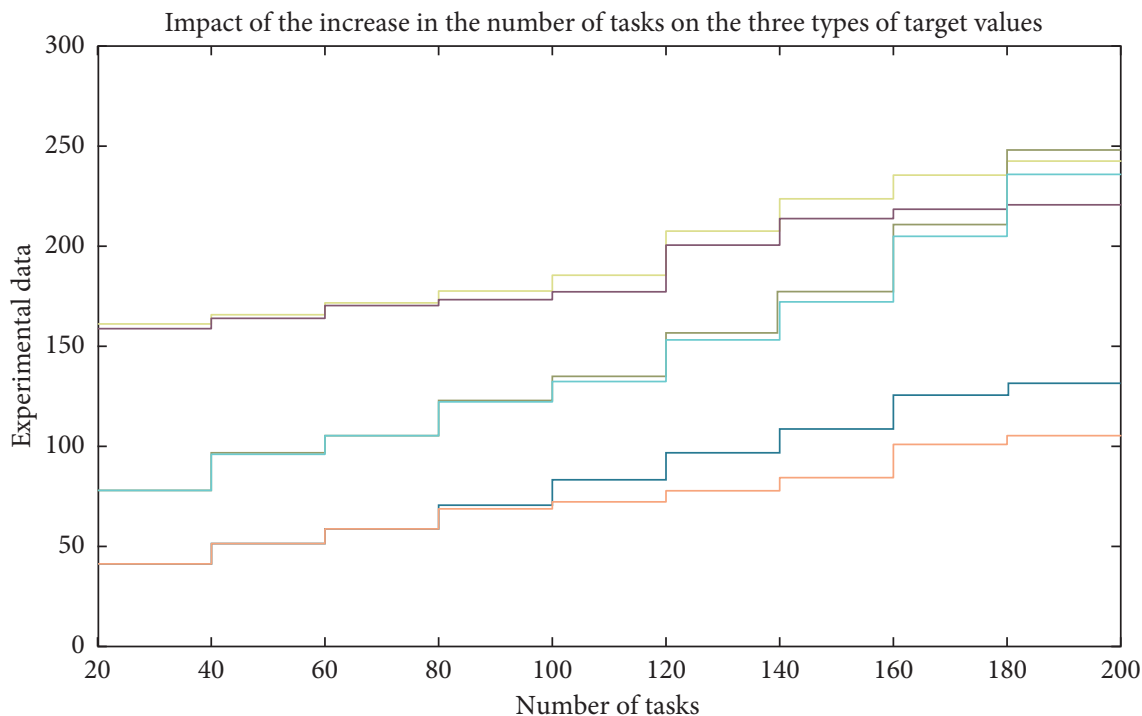
- Min-A
_ GA-B
GA-A
- Min-C
Min-B
_ GA-C

Figure 8: Impact of increase in the number of tasks on three types of target values. 




FIGURE 9: Comparison of crowded distance average of three algorithms.

\section{Conclusions}

This article analyzes the actual needs and business needs in current basketball training and puts forward functional requirements for the system based on these needs. Then, based on these functional requirements, the goal and technical route of the system are formulated, and the system is divided into acquisition and transmission. Four physical modules are terminal, data collection and calculation server, business analysis server, and physical fitness database. The physical fitness analysis system needs to complete data collection, including the data that the fitness analysis business may need to collect. The design also leaves a part of the data interface and business rule interface and isolates the data layer, calculation layer, model layer, and view layer through layered means, which retains the scalability for the expansion of physical business requirements. The development of the basketball training system in this paper not only realizes the expert-level vision of the demander, but also provides a lot of research on physical fitness analysis. It also involves studying and judging athletes' personal competition performance, analyzing athletes' personal training directions and plans, and comparing athletes horizontally. Athlete selection and role adjustment, adjustment of the overall training plan, verification of individual training theory, research and judgment of local competitive level, and selection of athlete talents have provided strong technical support for basketball training and teaching in my country.

This paper improves the traditional GA and establishes a fitness value calibration method to prevent a single fitness value from affecting the crowding distance, which ensures the uniformity of the population distribution and proposes an adaptive strategy based on nondominated sorting.

In the research process, due to various constraints, this article is still lacking in the consideration of some intelligent factors. Coupled with the lack of terminal development experience, the size of the test device is too large, which has a certain impact on the athletes. In future research, this article proposes to better improve the assistance of the training system based on dynamic intelligent fog computing in practical life from the following perspectives: (1) supplement high-end indicators to increase its coverage; (2) establish current training effects flexible functional relationship between indicators and field data; (3) there is still room for continued optimization of the fog computing resource management algorithm.

\section{Data Availability}

The data that support the findings of this study are available from the corresponding author upon reasonable request.

\section{Conflicts of Interest}

The author declares no conflicts of interest.

\section{References}

[1] M. A. Al Faruque and K. Vatanparvar, "Energy managementas-a-service over fog computing platform," IEEE Internet of Things Journal, vol. 3, no. 2, pp. 161-169, 2016.

[2] A. M. Rahmani, T. N. Gia, B. Negash et al., "Exploiting smart E-health gateways at the edge of healthcare internet-of-things: A fog computing Approach," Future Generation Computer Systems, vol. 78, no. 2, pp. 641-658, 2017.

[3] M. Peng, S. Yan, K. Zhang et al., "Fog computing based radio access networks: issues and challenges," IEEE Network, vol. 30, no. 4, pp. 46-53, 2015.

[4] W. Shi, J. Cao, Q. Zhang, Y. Li, and L. Xu, "Edge computing: vision and challenges," IEEE Internet of Things Journal, vol. 3, no. 5, pp. 637-646, 2016.

[5] M. Satyanarayanan, "The emergence of edge computing," Computer, vol. 50, no. 1, pp. 30-39, 2017.

[6] S. Ningning, G. Chao, A. Xingshuo, and Z. Qiang, "Fog computing dynamic load balancing mechanism based on graph repartitioning," China Communications, vol. 13, no. 3, pp. 156-164, 2016.

[7] A. V. Dastjerdi and R. Buyya, "Fog computing: Helping the internet of things realize its potential," Computer, vol. 49, no. 8, pp. 112-116, 2016.

[8] Z. Lv, D. Chen, R. Lou, and Q. Wang, "Intelligent edge computing based on machine learning for smart city," Future Generation Computer Systems, vol. 115, pp. 90-99, 2021.

[9] F. Jalali, K. Hinton, R. Ayre, T. Alpcan, and R. S. Tucker, "Fog computing may help to save energy in cloud computing," IEEE Journal on Selected Areas in Communications, vol. 34, no. 5, pp. 1728-1739, 2016.

[10] J. Ni, K. Zhang, X. Lin, and X. Shen, "Securing fog computing for internet of things applications: Challenges and solutions," IEEE Communications Surveys \& Tutorials, vol. 20, no. 99, pp. 601-628, 2018.

[11] S. Namasudra and P. Roy, "PpBAC: popularity based access control model for cloud computing," Journal of Organizational and End User Computing, vol. 30, no. 4, pp. 14-31, 2018.

[12] S. Sarkar and S. Misra, "Theoretical modelling of fog computing: a green computing paradigm to support IoT applications," IET Networks, vol. 5, no. 2, pp. 23-29, 2016.

[13] M. Elhoseny, "Multi-object detection and tracking (MODT) machine learning model for real-time video surveillance systems," Circuits, Systems, and Signal Processing, vol. 39, no. 2, pp. 611-630, 2019.

[14] X. Zhang, Y. Tian, and Y. Jin, "A knee point-driven evolutionary algorithm for many-objective optimization,” IEEE 
Transactions on Evolutionary Computation, vol. 19, no. 6, pp. 761-776, 2015.

[15] Y. Tsujimura, M. Gen, and E. Kubota, "Solving job-shop scheduling problem with fuzzy processing time using genetic algorithm," Journal of Japan Society for Fuzzy Theory \& Systems, vol. 7, no. 5, pp. 1073-1083, 2017.

[16] C. P. Mohanty, "Studies on some aspects of multi-objective optimization: a case study of electrical discharge machining process," vol. 146, no. 146, pp. 3295-3299, National Institute of Technology, Rourkela, India, 2015, Ph. D. thesis.

[17] A. M. Al-Momani, M. A. Mahmoud, and M. S. Ahmad, "Factors that influence the acceptance of internet of things services by customers of telecommunication companies in Jordan," Journal of Organizational and End User Computing, vol. 30, no. 4, pp. 51-63, 2018.

[18] V. Kozeny, "Genetic algorithms for credit scoring: alternative fitness function performance comparison," Expert Systems with Applications, vol. 42, no. 6, pp. 2998-3004, 2015.

[19] P. Guo, W. Cheng, and Y. Wang, "Hybrid evolutionary algorithm with extreme machine learning fitness function evaluation for two-stage capacitated facility location problems," Expert Systems with Applications, vol. 71, pp. 57-68, 2017.

[20] D. H. Al-Janan and T.-K. Liu, "Path optimization of CNC PCB drilling using hybrid Taguchi genetic algorithm," Kybernetes, vol. 45, no. 1, pp. 107-125, 2016.

[21] V. Pande, C. Marlecha, and S. Kayte, "A review-fog computing and its role in the Internet of Things," International Journal of Engineering Research and Applications, vol. 6, no. 10, pp. 2248-96227, 2016. 